\title{
Zuo Gui Wan Alters Expression of Energy Metabolism Genes and Prevents Cell Death in High-Glucose Loaded Mouse Embryos
}

\author{
Qi Liang $\mathbb{D}^{1}{ }^{1}$ Zhipeng Qu, ${ }^{2}$ Yu Liang, ${ }^{1}$ QianJin Feng, ${ }^{1}$ Xin Niu, ${ }^{3}$ Temaka Bai, ${ }^{3}$ \\ Yingli Wang, ${ }^{1}$ Qiang Song $\mathbb{D}^{1}{ }^{1}$ and David L. Adelson $\mathbb{D}^{2}$ \\ ${ }^{1}$ Shanxi University of Chinese Medicine, Taiyuan 030619, China \\ ${ }^{2}$ School of Biological Sciences, University of Adelaide, Adelaide, SA 5005, Australia \\ ${ }^{3}$ Beijing University of Chinese Medicine, Beijing 100029, China \\ Correspondence should be addressed to Qiang Song; 13834648844@163.com and David L. Adelson; david.adelson@adelaide.edu.au
}

Received 7 December 2017; Revised 15 March 2018; Accepted 3 April 2018; Published 25 June 2018

Academic Editor: Mohammed S. Razzaque

Copyright (c) 2018 Qi Liang et al. This is an open access article distributed under the Creative Commons Attribution License, which permits unrestricted use, distribution, and reproduction in any medium, provided the original work is properly cited.

Background. Zuo Gui Wan (ZGW) is a classic formula in traditional chinese medicine (TCM). Previous studies have shown that it is beneficial for impaired glucose tolerance (IGT) of adults and the offspring as well. This study aimed to understand the molecular mechanisms of the efficacy of ZGW on IGT. Methods. We used high-glucose loaded 2-cell stage mouse embryos as a model and took advantage of single-cell RNA sequencing technology to analyze the transcriptome of the model with or without ZGW. Differential gene expression analysis was performed with DESeq2. Results. High glucose can downregulate genes in the ribosome pathway, while ZGW can reverse this inhibition and as a result prevent embryo cell death caused by high glucose. Furthermore, high glucose can affect sugar metabolism and influence mitochondrial function, but ZGW can promote sugar metabolism via the tricarboxylic acid cycle mainly through upregulating the genes in the respiratory chain and oxidative phosphorylation. Conclusions. ZGW had a protective effect on embryonic cell death caused by glucose loading. The reversion of inhibition of ribosome pathway and regulation of mitochondrial energy metabolism are main effects of ZGW on high-glucose loaded embryos. This research not only revealed the global gene regulation changes of high glucose affecting 2-cell stage embryos but also provided insight into the potential molecular mechanisms of ZGW on the IGT model.

\section{Background}

Impaired glucose tolerance (IGT) is a sugar metabolism disorder between normal glucose tolerance (NGT) and diabetes mellitus [1]. It is estimated that there are 308 million people with IGT in the world [2], many more than those diagnosed with diabetes. People with IGT can progress to $\mathrm{DM}$ and are predisposed to cardiocerebrovascular disease [3-8], microvascular disease [9], lipid metabolism disorders [10], and chronic kidney disease [11, 12]. Therefore, it is becoming more and more important to understand how to prevent IGT. Currently, drugs such as Metformin, Acarbose, and Rosiglitazone are used to treat IGT and can postpone the occurrence of diabetes; however, they cannot prevent corresponding complications [13-18]. Many reports have shown that TCM might be one of the resources to develop new methods for preventing IGT [19-25].

In a previous report, three different Chinese formulas, Zuo Gui Wan (ZGW), You Gui Wan (YGW), and Ba Zhen Tang (BZD), were separately used to treat pregnant Wistar rats. After drugs were administered for three weeks, offspring from different groups were then fed with a high-fat diet for 12 weeks. Many indices, such as fasting blood glucose (FBG), 2-hour blood glucose (2hBG), blood lipid, fasting serum insulin (FINS), and leptin and adiponectin (APN), were measured. Results showed that rats on a high-fat diet developed IGT with abnormal blood lipid, insulin resistance, leptin resistance, and fatty liver. However, ZGW could prevent IGT in these offspring. Compared to YGW and BZD, which only reversed part of above indexes, ZGW 
was the most effective formula [26]. Another recent report has also found that giving ZGW to Gestational Diabetes Mellitus (GDM) rats can have a preventive effect on the IGT of offspring induced by a high-fat and high-sugar diet [27]. ZGW is a classic traditional Chinese medicine (TCM) formula with extract from 8 traditional Chinese medicines, which are Rehmannia glutinosa (Shu Di Huang), Cuscuta chinensis (Tu Si Zi), Cornus officinalis (Shan Zhu Yu), Lycium barbarum (Gou Qi Zi), Dioscorea opposita (Shan Yao), Cyathula officinalis (Chuan Niu Xi), Cervi cornus Colla (Lu Jiao Jiao), and Chinemys reevesii (Gui Ban Jiao). 12 main metabolites were detected in ZGW rat serum with UPLC/MS: $\beta$-D-ribofuranuronic acid methyl ester triacetate, 5-hydroxymethyl-2-furfural glucuronide, dihydro-5-hydroxymethyl-2-furfural glucuronide, 8-epiloganic acid, loganic acid, morroniside, coumaric acid, loganin, sweroside, 3hydroxy-2,6,6-trimethyl-1-cyclohexene-1-carboxylic acid, kaempferol-3-glucuronide, and cuscutamine. Of these, morroniside and loganin could regulate rat mesangial cell growth by reducing oxidative stress and could be used at the early stages of diabetic nephropathy [28]. Studies have shown that dietary kaempferol may reduce the risk of chronic diseases, especially cancers, by augmenting antioxidants to combat free radicals [29].

Previous studies have shown that ZGW is an effective treatment in the IGT rat model, and it is beneficial not only to the mother but also to the offspring [26, 27, 30]. But the molecular mechanisms of ZGW on IGT, particularly at the transcriptome level, are still unclear. Therefore, in this study, we used mouse embryos loaded with high glucose as an IGT model to study the effect of ZGW. By analyzing the transcriptome of our IGT model treated with ZGW, we identified ribosome pathway and oxidative phosphorylation as the potential target molecular pathways of ZGW on IGT. A list of potential response genes to ZGW on IGT was also identified, and these genes provide a good resource for further functional studies.

\section{Methods}

All chemicals used in this study were purchased from SigmaAldrich Corporation (St. Louis, MO, USA) unless otherwise indicated. The Institutional Animal Care and Use Committee at the China Agricultural University (Beijing, China) approved the protocols used in this study.

2.1. Preparation of $Z G W$. ZGW is a classic formula in TCM and includes the extract from 8 traditional Chinese medicines. First, these 8 medicines were immersed in $800 \mathrm{ml}$ water at $50^{\circ} \mathrm{C}$ and then decocted for 1.5 hours and filtered. The decoction and filtration were repeated two times. The filtrates were combined and concentrated to $1 \mathrm{~g} / \mathrm{ml}$ crude drug.

2.2. ZGW Serum Preparation. Rats from National Institutes for Food and Drug Control, China (license number: SCXK(JING) 2009- 0017), were fed with $20 \mathrm{~g} / \mathrm{kg} / \mathrm{d}$ ZGW for 7 days. Blood was collected directly from their hearts and incubated at $4^{\circ} \mathrm{C}$ for $30 \mathrm{~min}$, followed by centrifugation at $4000 \mathrm{rpm}$ for $15 \mathrm{~min}$ at $4^{\circ} \mathrm{C}$. The serum, denoted as $\mathrm{ZGW}$-containing rat serum, was collected immediately and stored at $-75^{\circ} \mathrm{C}$ before use. The concentration of ZGW rat serum used in this research was $0.01 \% \mathrm{v} / \mathrm{v} \mathrm{ZGW}$.

2.3. Super Ovulation. For timed pregnancy, pregnant mare's serum gonadotropin (PMSG) (5 IU) was intraperitoneally injected into ICR female mice aged 6 to 8 weeks. Next, human chorionic gonadotropin (hCG) (5 IU) was intraperitoneally injected after $48 \mathrm{~h}$; on the evening of hCG injection, male mice and the female mice $(2: 1)$ were housed together in a cage for one night. The next morning, females were checked for a vaginal plug to determine if they were pregnant.

2.4. Drug Administration and Grouping. Pregnant mice were cervically dislocated and zygotes were washed out from the vagina. All zygotes were randomly assigned to three groups: control group, model group, and drug group. Each group contained 9 zygotes. Zygotes in different groups were cultured with media as follows: control group with cellculture medium, model group with cell culture medium supplemented with high glucose $15.6 \mathrm{mmol} / \mathrm{L}$, and drug group similar to the model group but with the addition of rat serum containing $0.01 \% \mathrm{v} / \mathrm{v}$ ZGW.

2.5. Determination of Blastocyst Embryo Cell Number. Zygotes in the three groups were cultured in vitro for five days (blastocyst stage) and then incubated separately in $\mathrm{M} 2$ medium $\left[\mathrm{NaCl}(5.533 \mathrm{~g} / \mathrm{L}), \mathrm{KCl}(0.356 \mathrm{~g} / \mathrm{L}), \mathrm{CaCl}_{2} \cdot 2 \mathrm{H}_{2} \mathrm{O}\right.$ $(0.252 \mathrm{~g} / \mathrm{L}), \mathrm{KH}_{2} \mathrm{PO}_{4}(0.162 \mathrm{~g} / \mathrm{L}), \mathrm{MgSO}_{4} \cdot 7 \mathrm{H}_{2} \mathrm{O}(0.293 \mathrm{~g} / \mathrm{L})$, $\mathrm{NaHCO}_{3}(0.349 \mathrm{~g} / \mathrm{L})$, Hepes $(4.969 \mathrm{~g} / \mathrm{L})$, sodium lactate $(2.610 \mathrm{~g} / \mathrm{L})$, sodium pyruvate $(0.036 \mathrm{~g} / \mathrm{L})$, glucose $(1.000 \mathrm{~g} / \mathrm{L})$, BSA $(4.000 \mathrm{~g} / \mathrm{L})$, penicillin $(0.060 \mathrm{~g} / \mathrm{L})$, and streptomycin $(0.050 \mathrm{~g} / \mathrm{L})]$ containing Hoechst $33342(10 \mu \mathrm{g} / \mathrm{mL})$ for 15 minutes at $37^{\circ} \mathrm{C}$. After washing three times with M2 medium, blastocysts from the three groups were separately mounted on microscope slides and examined on an epifluorescence microscope to count the number of embryo cell nuclei.

2.6. Sample Preparation for Single-Cell RNA Sequencing. Zygotes from the three groups were cultured in vitro to 2cell stage, and three zygotes were randomly selected as a sample from each group and stored in liquid nitrogen. Each group was replicated three times for biological replication. Zygotes were resuspended in freshly prepared lysis buffer [total volume $100 \mu \mathrm{l}$, containing $93 \mu \mathrm{l}$ nuclease-free water, $2 \mu$ ] Triton X-100, and $5 \mu \mathrm{l}$ RNaseOUT $(20 \mathrm{U} / \mu \mathrm{l})$ ]; each zygote was lysed with a micropipette to yield more than $7 \mu$ l of lysate and the lysate stored at $-80^{\circ} \mathrm{C}$.

2.7. Single-Cell RNA Sequencing and Bioinformatic Analysis. RNA amplification was performed according to SMARTer Ultra Low Input RNA for Illumina Kit (Clontech Laboratories). Quantity and quality of amplified cDNAs were measured with Qubit and Agilent Bioanalyzer 2100 (Agilent Technologies, Santa Clara, CA, USA). RNA sequencing was performed using Illumina HiSeq 2500 (Shanghai Biotechnology Corporation, Shanghai, China). Adaptors and lowquality sequences from raw reads were filtered using Trim Galore! with the following parameter: stringency 6 . Trimmed 


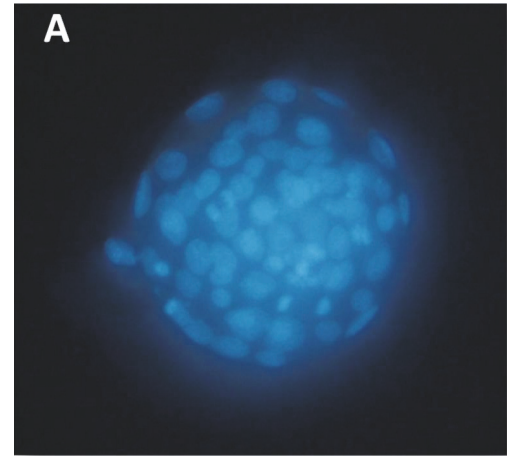

(a)

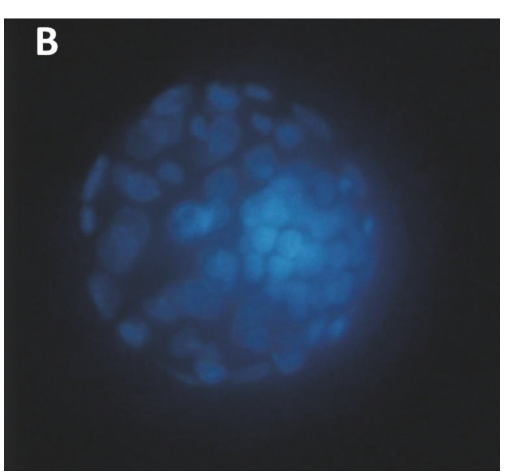

(b)

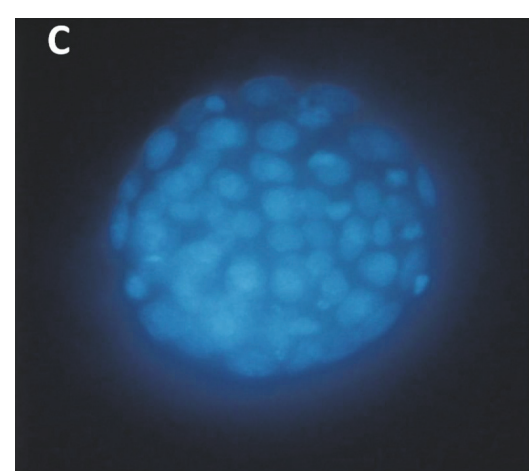

(c)

FIGURE 1: Fluorescence photomicrographs of blastocyst cells stained with Hoechst 33342. (a) Stained blastocyst cells in control group. (b) Stained blastocyst cells in model group. (c) Stained blastocyst cells in drug group.

TABLE 1: Effects of ZGW rat serum on blastocyst cell number.

\begin{tabular}{lc}
\hline Group & Blastocyst cell number $(n)$ \\
\hline Control & $68.4^{\mathrm{a}} \pm 2.4$ \\
Model & $57.2^{\mathrm{a}, \mathrm{b}} \pm 1.6$ \\
Drug & $63.2^{\mathrm{b}} \pm 2.2$ \\
\hline
\end{tabular}

Values are mean \pm SEM. Means in column with different superscripts indicate significant differences $(P<0.05)$.

reads were mapped to the mouse reference genome (mm10) using STAR v2.5 with the following parameters: out Filter Mismatch Nover $L$ max 0.05 and seed Search Start $L$ max 30 [31]. Raw counts of mouse RefSeq genes were acquired with R package "Genomic Alignments" [32]. Differential gene expression analysis was performed with DESeq2 [33]. Gene Ontology (GO) and Kyoto Encyclopedia of Genes and Genomes (KEGG) enrichment analyses were performed using Clue GO based on significantly differentially expressed genes (false discovery rate $<0.05$ ) from DESeq2 [1]. Enriched GO terms or KEGG pathways were visualized with Cytoscape V3 [34].

\section{Results}

3.1. Blastocyst Cell Number. In the control group (Figure 1(a)), the cells in blastocyst showed bright nuclear fluorescence. But in the model group (Figure 1(b)), blastocysts showed decreases in both nuclear fluorescence and the number of nuclei. In contrast to this, in the drug group (Figure 1(b)), both nuclear fluorescence and the number of nuclei in blastocysts increased compared to the model group. There were significant differences in the blastocyst cell number between the control group and the model group $(P<0.05)$ and between the model group and the drug group $(P<0.05)$ (Table 1).

3.2. Summary of Transcriptome Analysis. Principal component analysis (PCA) was able to resolve and separate the three groups (Figure 2), indicating overall differences in the

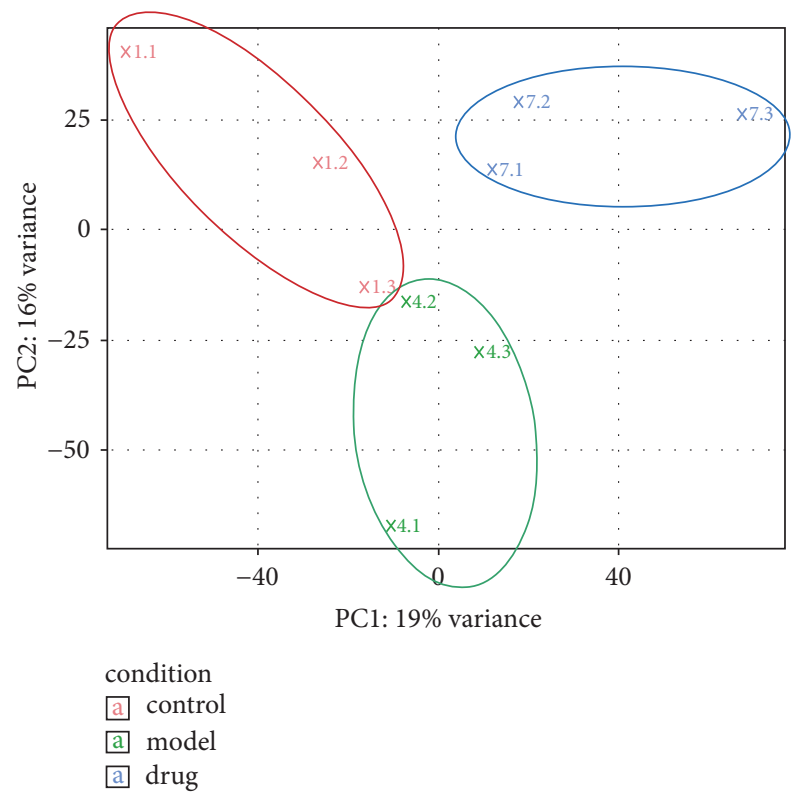

FIGURE 2: PCA of gene expression patterns for 2-cell stage embryos. Different colors identify different groups as indicated in the legend.

patterns of gene expression in these treatments. The $M A$ plot (" $M$ " is for log ratios and " $A$ " is for mean average) (Figure 3) demonstrates that most of the points were clustered tightly around the horizontal line, indicating that RNA-Seq data were of good quality. There were differently expressed genes between these three groups (red dots), indicating that expression of some genes was changed after the control group was loaded with high glucose (Figure 3(a)) and after the model group was treated with ZGW rat serum (Figure 3(b)). Furthermore, by comparing the global gene expression changes between model group and control group, we identified 71 upregulated and 100 downregulated genes, respectively. We then also compared the drug group to the model group, and 115 upregulated and 174 downregulated genes were detected (Table 2). 


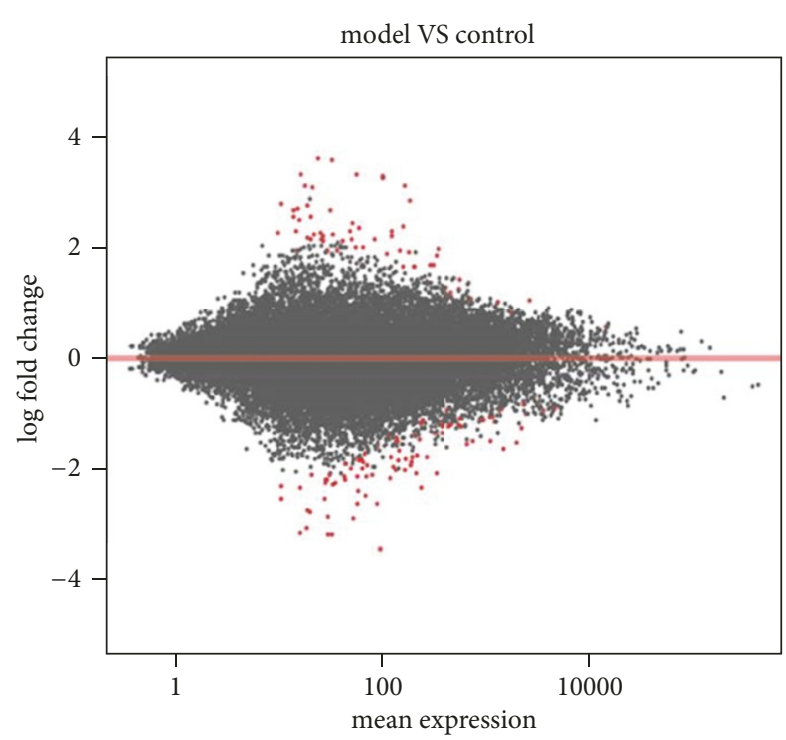

(a)

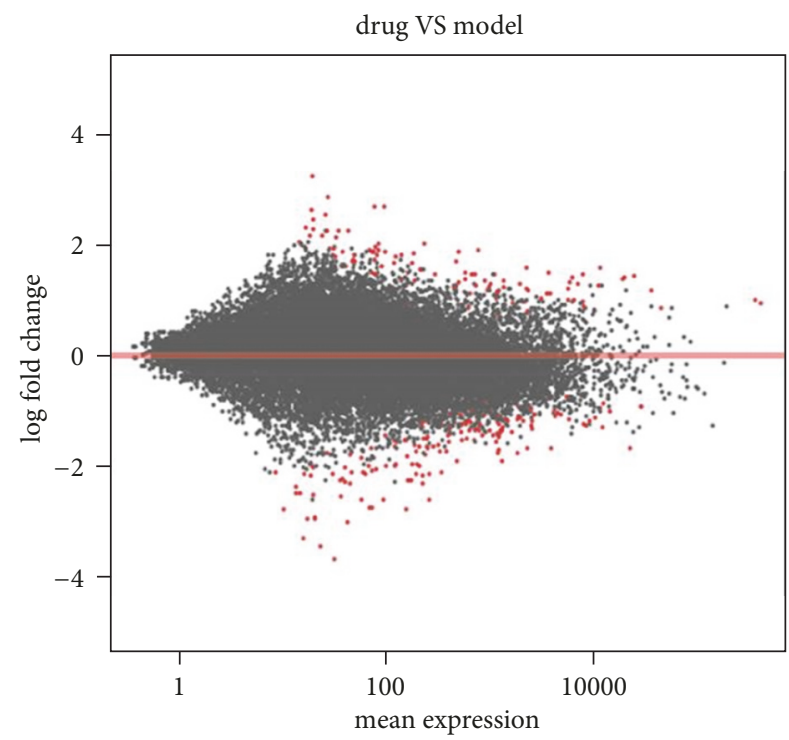

(b)

FiguRE 3: MA-plot of the $\log 2$ fold changes over the mean of RNA-Seq read counts. The $\log 2$ fold change for a particular comparison is plotted on $y$-axis and the average of the counts normalized by size factor is shown on $x$-axis. Each gene is represented with a dot, and genes with FDR $<0.05$ are shown in red. (a) Comparison of model group with control group. (b) Comparison of drug group with model group.

TABLE 2: Summary of differentially regulated gene numbers among the three groups $(\mathrm{FDR}<0.05)$.

\begin{tabular}{lccc}
\hline & Control & Model & Drug \\
\hline Control & N/A & $\mathbf{1 0 0}$ & N/A \\
Model & 71 & N/A & $\mathbf{1 7 4}$ \\
Drug & N/A & 115 & N/A \\
\hline
\end{tabular}

Numbers in italic are upregulated genes, while those in bold are downregulated (DOCX).

3.3. Pathway Analysis of Embryos Treated with High Glucose. To identify the gene regulatory pathways potentially affected when embryos were treated with high glucose, two gene enrichment analyses were carried out for the differentially expressed genes between the model and control groups. For the Gene Ontology (GO) enrichment analysis (Table 3), we found 6 enriched biological process GO terms, including "gluconeogenesis" (GO: 0006094). For cellular components, we found ribosome (GO: 0022625, GO: 0015934, and GO: 0022626) and mitochondrial respiratory chain (GO: 0005746) as overrepresented terms. The insulin-like growth factor binding (GO: 0005520) was identified as the most significant overrepresented GO term in the molecular function category. Based on KEGG enrichment analysis, ribosome was identified as the only significantly overrepresented pathway with differentially expressed genes enriched in 2-cell stage of mouse embryo treated with high glucose (Figure 4).

3.4. Gene Expression Changes in High-Glucose Loaded Embryos Treated with $Z G W$ Rat Serum. We used the same enrichment analysis to analyze the potential molecular

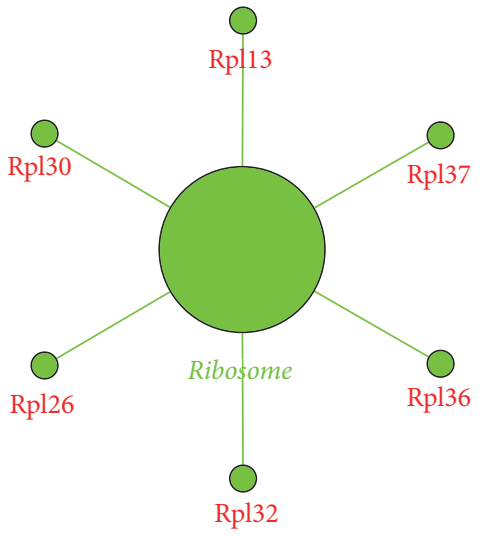

FIGURE 4: KEGG pathway (FDR < 0.05) enriched by the 171 differentially expressed genes in model group compared to control group. Green colored dots are downregulated. The big dot is a sign of the enriched pathway and the big green word is the name of the pathway. Small green dots show the genes that are downregulated, and the red words are the names of downregulated genes.

pathways affected by ZGW in high-glucose loaded embryos. Based on differentially expressed genes in the drug group compared to the model group, 22 overrepresented GO terms were observed and 13 of them were in the molecular function category (Table 4). These terms included hydrogen ion transmembrane transporter activity (GO: 0015078), cytochrome-c oxidase activity (GO: 0004129), heme-copper terminal oxidase activity (GO: 0015002), and oxidoreductase activity (GO: 0016676, GO: 0016675). For cellular component terms, we found two main locations, the ribosome and mitochondria, with terms such as ribosomal subunit (GO: 
TABLE 3: Summary of GO terms for differentially expressed genes between model and control groups.

\begin{tabular}{|c|c|c|c|}
\hline Ontology source & GO ID & GO term & Term $P$ value \\
\hline \multirow{6}{*}{ Biological process } & GO: 0034616 & Response to laminar fluid shear stress & $1.07 E-04$ \\
\hline & GO: 0006525 & Arginine metabolic process & $4.12 E-04$ \\
\hline & GO: 0034405 & Response to fluid shear stress & $2.18 E-03$ \\
\hline & GO: 0021885 & Forebrain cell migration & $1.63 E-02$ \\
\hline & GO: 0035914 & Skeletal muscle cell differentiation & $1.75 E-02$ \\
\hline & GO: 0006094 & Gluconeogenesis & $1.81 E-02$ \\
\hline \multirow{6}{*}{ Cellular component } & GO: 0022625 & Cytosolic large ribosomal subunit & $1.19 E-05$ \\
\hline & GO: 0015934 & Large ribosomal subunit & $1.53 E-04$ \\
\hline & GO: 0022626 & Sytosolic ribosome & $2.36 E-04$ \\
\hline & GO: 0031941 & Filamentous actin & $1.19 E-03$ \\
\hline & GO: 0005881 & Cytoplasmic microtubule & $8.66 E-03$ \\
\hline & GO: 0005746 & Mitochondrial respiratory chain & $1.18 E-02$ \\
\hline \multirow{3}{*}{ Molecular function } & GO: 0005520 & Insulin-like growth factor binding & $6.88 E-04$ \\
\hline & GO: 0016879 & Ligase activity, forming carbon-nitrogen bonds & $7.94 E-03$ \\
\hline & GO: 0033613 & Activating transcription factor binding & $9.60 E-03$ \\
\hline
\end{tabular}

15 GO terms are significantly enriched by the 171 changed genes after control group loaded by high glucose.

TABLE 4: Summary of GO terms for differentially expressed genes between drug and model groups.

\begin{tabular}{|c|c|c|c|}
\hline Ontology source & GO ID & GO term & Term $P$ value \\
\hline \multirow{2}{*}{ Biological process } & GO: 1902600 & Hydrogen ion transmembrane transport & $2.60 E-04$ \\
\hline & GO: 0070646 & Protein modification by small protein removal & $6.99 E-04$ \\
\hline \multirow{7}{*}{ Cellular component } & GO: 0005732 & Small nucleolar ribonucleoprotein complex & $3.34 E-06$ \\
\hline & GO: 0005685 & U1 snRNP & $1.47 E-03$ \\
\hline & GO: 0044391 & Ribosomal subunit & $1.52 E-03$ \\
\hline & GO: 0005753 & Mitochondrial proton-transporting ATP synthase complex & $1.70 E-03$ \\
\hline & GO: 0045259 & Proton-transporting ATP synthase complex & $2.52 E-03$ \\
\hline & GO: 0016469 & Proton-transporting two-sector ATPase complex & $2.57 E-03$ \\
\hline & GO: 0044455 & Mitochondrial membrane part & $3.11 E-03$ \\
\hline \multirow{13}{*}{ Molecular function } & GO: 0015078 & Hydrogen ion transmembrane transporter activity & $1.90 E-04$ \\
\hline & GO: 0004129 & Cytochrome-c oxidase activity & $4.00 E-04$ \\
\hline & GO: 0015002 & Heme-copper terminal oxidase activity & $4.00 E-04$ \\
\hline & GO: 0016676 & $\begin{array}{l}\text { Oxidoreductase activity, acting on a heme group of donors, } \\
\text { oxygen as acceptor }\end{array}$ & $4.00 E-04$ \\
\hline & GO: 0008553 & $\begin{array}{l}\text { Hydrogen-exporting ATPase activity, phosphorylative } \\
\text { mechanism }\end{array}$ & $4.19 E-04$ \\
\hline & GO: 0016675 & Oxidoreductase activity, acting on a heme group of donors & $4.55 E-04$ \\
\hline & GO: 0030515 & snoRNA binding & $3.03 E-03$ \\
\hline & GO: 0036442 & Hydrogen-exporting ATPase activity & $3.79 E-03$ \\
\hline & GO: 0016830 & Carbon-carbon lyase activity & $3.95 E-03$ \\
\hline & GO: 0016814 & $\begin{array}{l}\text { Hydrolase activity, acting on carbon-nitrogen (but not } \\
\text { peptide) bonds, in cyclic amidines }\end{array}$ & $4.66 E-03$ \\
\hline & GO: 0000062 & Fatty-acyl-CoA binding & $6.17 E-03$ \\
\hline & GO: 0019783 & Ubiquitin-like protein-specific protease activity & $6.26 E-03$ \\
\hline & GO: 0019843 & rRNA binding & $3.21 E-02$ \\
\hline
\end{tabular}

22 GO terms are significantly enriched by the 289 changed genes after model group treated with ZGW rat serum. 


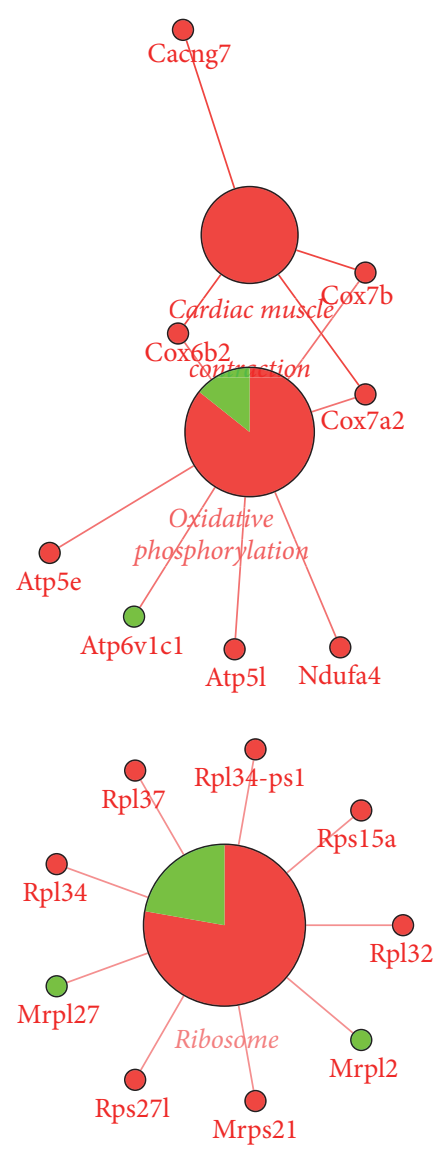

FIGURE 5: KEGG pathway (FDR < 0.05) enriched by the 289 differentially expressed genes in drug group compared to model group. Red means upregulated, while green means downregulated. Big dots represent the enriched pathways and big red words are the names of these pathways. Small red dots show the upregulated genes and the related red words are downregulated gene names. Small green dots show the downregulated genes and the related green words are downregulated gene names.

0044391), small nucleolar ribonucleoprotein complex (GO: 0005732), mitochondrial proton-transporting ATP synthase complex (GO: 0005753), and mitochondrial membrane part (GO: 0044455). Results of KEGG enrichment analysis showed that genes in the ribosome pathway were upregulated, and some genes in the oxidative phosphorylation pathway were also upregulated by ZGW (Figure 5).

3.5. Identification of Potential Response Genes for ZGW. Among the 71 upregulated genes in the model group compared to the control group, 14 genes were downregulated in embryos treated with ZGW (Figure 6). These genes were Irf6, H2afy, Smarcal, Fmrlos, Ifi27, Fam46c, Cym, Caps2, Rassf8, Xkr4, Plac1, Xrra1, 3110001I22Rik, and A230056J06Rik (Figure 7). No GO terms could be enriched based on these 14 genes. In the 100 downregulated genes from the model group compared to the control group, 14 genes were upregulated in embryos treated with ZGW (Figure 6). They were Krt27, Deb1, Myg1, Pmvk, Rpl37, Snord72, Gpalpp1, Zscan26, Rpl32, Adh1,
Slc17a1, Aurkaip1, Nop10, and Cox7b(Figure 8). These 14 genes were enriched for GO terms highly related to mitochondrial energy metabolism (Table 5).

\section{Discussion}

4.1. Effects of High Glucose on Mice Embryo Development. Ample studies have shown that high glucose can affect embryos and block embryo development [35-37]. Research on the mechanism of this embryo development block has shown that high glucose can induce reactive oxygen species and cause damage to the embryo through oxidative stress [37, 38]. In this study, we found that high glucose downregulated several genes in the ribosome pathway (Figure 4), and three GO terms of ribosome have been enriched based on the differentially expressed genes between the model and control groups. Ribosome mediates protein synthesis and the downregulation of genes involved in the ribosome pathway in high-glucose loaded embryos indicated that protein synthesis might be affected. Furthermore, 2-cell stage is a key stage between maternal regulation and zygotic regulation in mouse [39]. Downregulation of ribosomal genes at this stage could affect the regulation of this transition.

We also found that genes involved in gluconeogenesis, including $c r t c 2$, Ppara, and $S d s$, were all downregulated in the model group. Molecular function of "insulin-like growth factor binding (GO:0005520)" was also affected by high glucose (Table 3). Insulin growth factors (IGFs) are important proteins in the regulation of embryo development, and a study showed that IGFs were main endocrine factors in the regulation of embryo development [40]; IGF-I has effects on metabolic regulation in embryos [41, 42]. The gluconeogenesis process is one of the pathways regulated by sugar in the uterus [40].

All these indicated that downregulating genes in ribosome pathway and affecting the sugar metabolism of mice embryo at 2-cell stage are two of the main causes of embryo developmental block induced by high glucose.

4.2. Efficacy of ZGW on High-Glucose Loaded Mice Embryo. Previous studies showed that ZGW rat serum could induce cell proliferation and differentiation [43, 44], inhibit cell apoptosis [45], and promote germ cell and embryo development $[30,46]$. Furthermore, it has also been shown that the administration of ZGW to GDM rats has a preventive effect on the offspring's IGT caused by a high-fat and highsugar diet [27]. In our study, we found that ZGW could upregulate several genes in the ribosome pathway, which were downregulated in embryos loaded with high glucose (Figure 5). The ribosomal subunit (GO: 0044391) was also one of the terms that been affected by ZGW (Table 4). In addition, among the 14 downregulated genes in the model group but upregulated in the drug group (Figure 8), Rpl32 and Rpl37 are both involved in the ribosome pathway.

Furthermore, we found that some pathways associated with energy metabolism were altered in high-glucose loaded embryos after treatment with ZGW. ZGW upregulated genes in the oxidative phosphorylation pathway (Figure 5), consistent with activate energy metabolism and sugar metabolism 


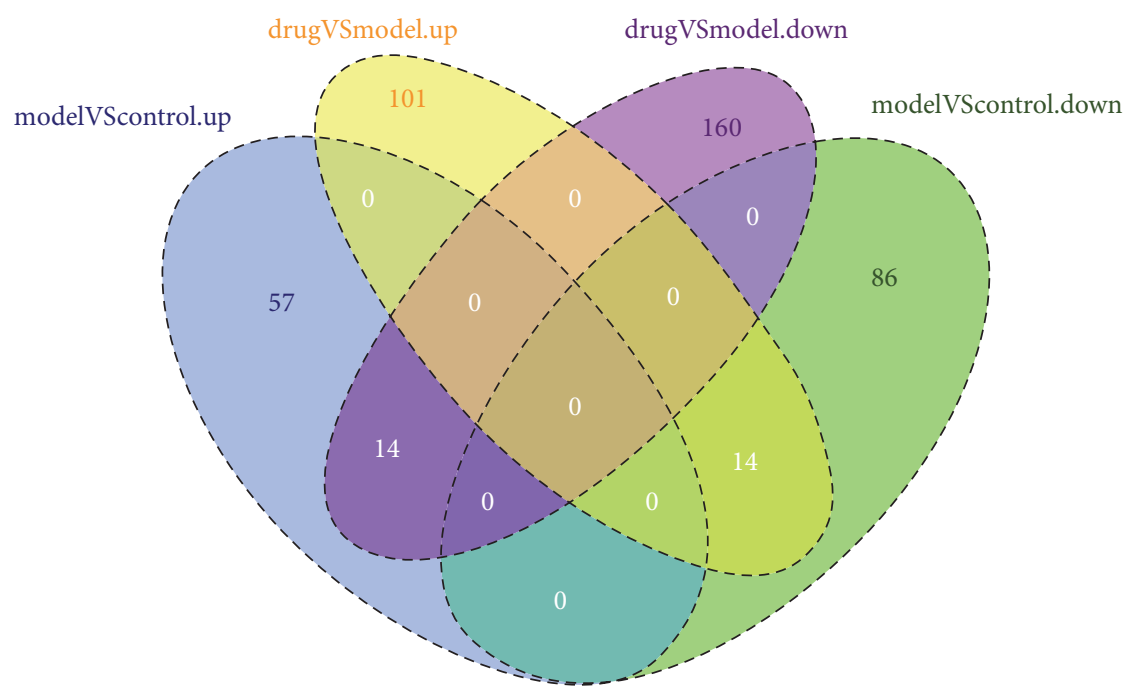

FIGURE 6: Venn diagram of pairwise gene expression changes between the three groups. Numbers in ovals represent different gene numbers between two groups.
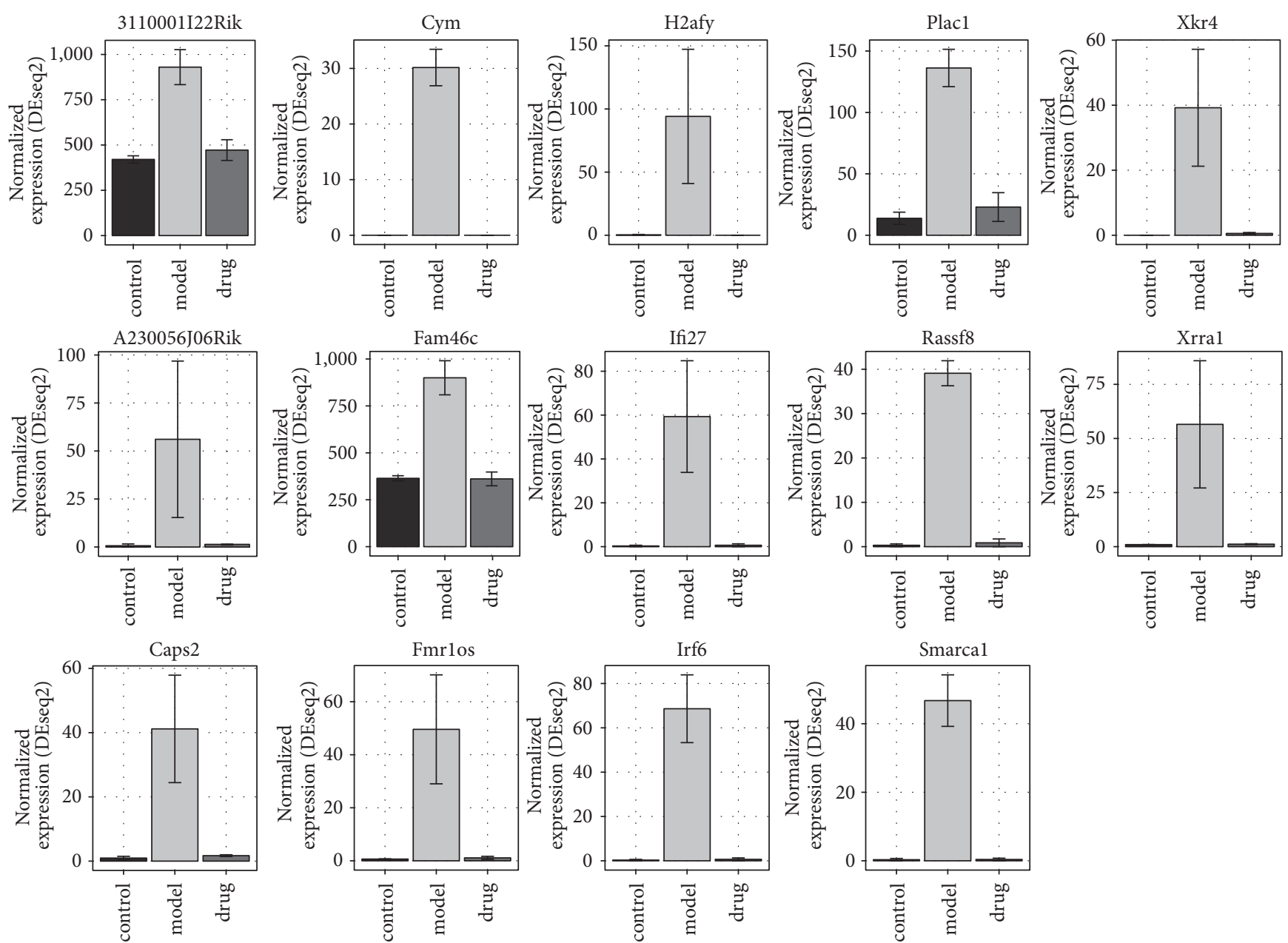

FIGURE 7: 14 genes upregulated in model group and downregulated in drug group. Read counts normalized by library size according to DESeq2 are shown on $y$-axis. 
TABLE 5: Summary of GO terms of 14 genes downregulated in model group and upregulated in drug group.

\begin{tabular}{lcr}
\hline GO ID & Description & $q$-value \\
\hline GO: 0005743 & Mitochondrial inner membrane & $1.92 E-06$ \\
GO: 0019866 & Organelle inner membrane & $2.07 E-06$ \\
GO: 0005746 & Mitochondrial respiratory chain & $2.19 E-05$ \\
GO: 0070469 & Respiratory chain & $2.19 E-05$ \\
GO: 0044455 & Mitochondrial membrane part & $6.72 E-05$ \\
GO: 1990204 & Oxidoreductase complex & $1.54 E-04$ \\
GO: 0030964 & NADH dehydrogenase complex & $4.25 E-04$ \\
GO: 0005747 & Mitochondrial respiratory chain complex I & $4.25 E-04$ \\
GO: 0045271 & Respiratory chain complex I & $4.25 E-04$ \\
\hline
\end{tabular}

9 GO terms are significantly enriched by the 14 reversed genes in drug group.
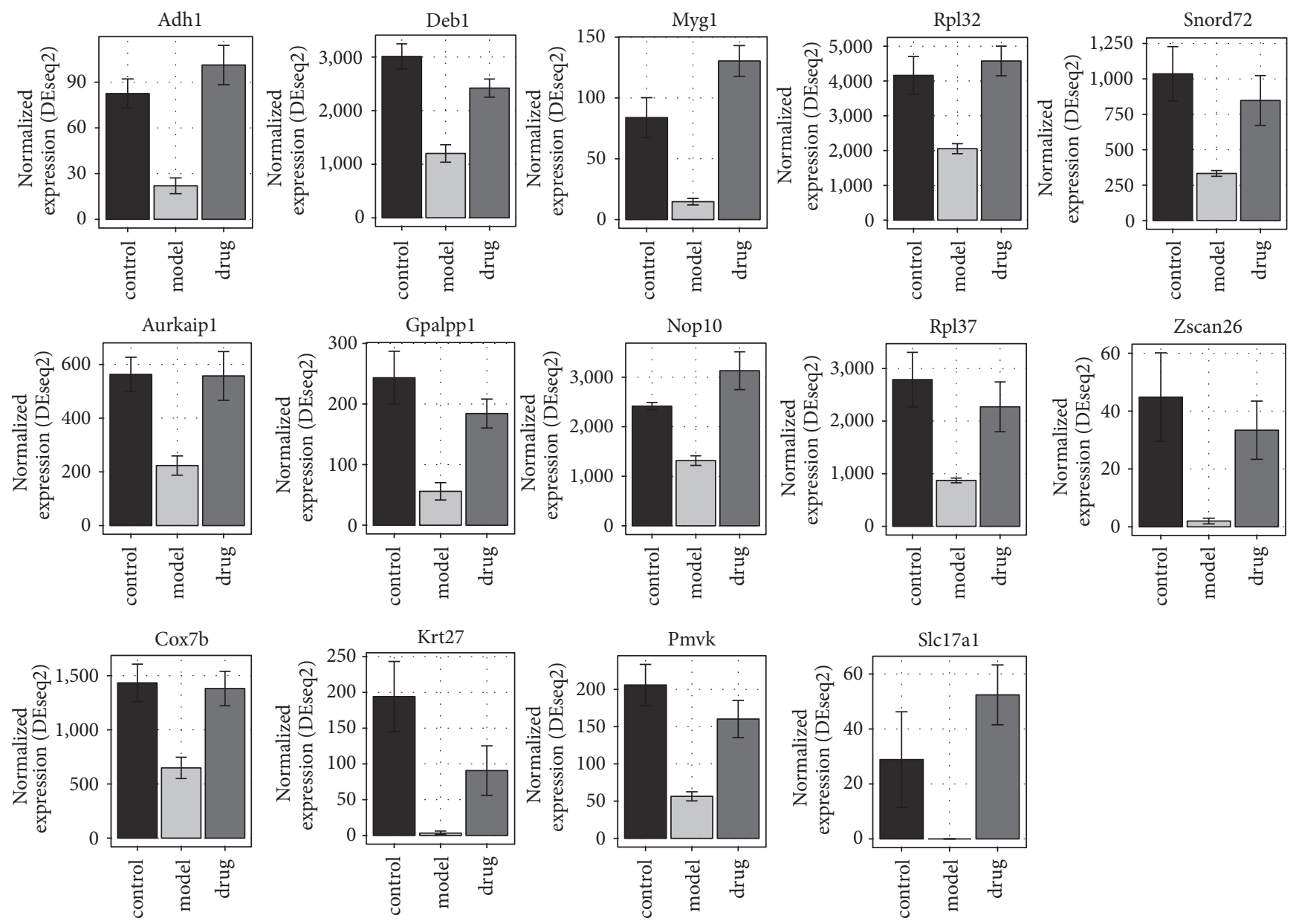

FIGURE 8: 14 genes downregulated in model group and upregulated in drug group. Read counts normalized by library size according to DESeq2 are shown on $y$-axis.

in embryos. Oxidoreductase activity (GO: 0016676 and GO: 0016675), mitochondrial proton-transporting ATP synthase complex (GO: 0005753), and mitochondrial membrane part (GO: 0044455) were also enriched based on the differentially expressed genes between the drug and model groups (Table 4). The GO annotation and coexpression network of 14 genes downregulated in the model group but upregulated by
ZGW in the drug group (Table 5) showed that some of them were associated with the mitochondrial energy metabolism.

By these results, taken together with the result showing that ZGW prevented the embryo cell death caused by high glucose and promotes the blastocyst formation rate and total cell numbers in blastocysts, we conclude that ZGW may reverse the inhibition of the ribosome pathway and increase 
mitochondrial energy metabolism, which were inhibited by high glucose, and prevent the mouse embryo cell death caused by high glucose.

\section{Conclusions}

IGT can be effectively modeled using mouse embryos loaded with high glucose, providing an effective means to explore its pathogenesis and molecular mechanisms. By analyzing the transcriptome of this IGT model treated with ZGW, we found that high glucose might affect sugar metabolism and influence the mitochondrial function of mouse embryos at the 2cell stage and that ZGW can counteract this by upregulating genes in the respiratory chain and oxidative phosphorylation. Furthermore, ZGW can prevent the embryo cell death caused by high glucose through upregulating genes inhibited by high glucose in ribosome pathway.

\section{Abbreviations}

\author{
ZGW: Zuo Gui Wan \\ TCM: Traditional Chinese medicine \\ IGT: Impaired glucose tolerance \\ NGT: Normal glucose tolerance \\ DM: Diabetes mellitus \\ YGW: You Gui Wan \\ BZD: Ba Zhen Tang \\ FBG: Fasting blood glucose \\ 2hBG: 2-hour blood glucose \\ FINS: Fasting serum insulin \\ APN: Leptin and adiponectin \\ GDM: Gestational Diabetes Mellitus \\ PMSG: Pregnant mare's serum gonadotropin \\ hCG: Human chorionic gonadotropin \\ PCA: Principal component analysis \\ GO: Gene Ontology enrichment analysis
}

KEGG: Kyoto Encyclopedia of Genes and Genomes.

\section{Data Availability}

All data are contained within the article. Raw data and material are available at https://www.ncbi.nlm.nih.gov/geo/query/acc .cgi? acc $=$ GSE85477.

\section{Ethical Approval}

The Institutional Animal Care and Use Committee at the China Agricultural University (Beijing, China) approved the protocols used in this study.

\section{Conflicts of Interest}

The authors declare that they have no conflicts of interest.

\section{Authors' Contributions}

Qi Liang, QianJin Feng, David L. Adelson, Qiang Song, and Xin Niu conceived and designed the experiments. Qi
Liang, Zhipeng Qu, and David L. Adelson performed the experiments and analyzed the transcriptome data. Qi Liang, Zhipeng Qu, Yu Liang, and David L. Adelson wrote the paper. Temaka Bai cultured the embryos and quantified blastocyst cells. Yingli Wang helped with the extraction of serum containing ZGW and the analysis of main metabolites in $\mathrm{ZGW}$ rat serum.

\section{Acknowledgments}

This study was funded by the Shanxi Province Key Research and Development Project (International Cooperation): the regulatory function of tonifying SHEN formula in TCM on the early development of mouse embryo at transcriptome level (no. 201703D421017). The authors wish to thank Zhu Shien of the National Engineering Laboratory for Animal Breeding of China Agricultural University (Beijing, China) for providing embryo culture technology.

\section{References}

[1] G. Bindea, B. Mlecnik, H. Hackl et al., "ClueGO: a Cytoscape plug-in to decipher functionally grouped gene ontology and pathway annotation networks," Bioinformatics, vol. 25, no. 8, pp. 1091-1093, 2009.

[2] S. J. Grant, A. Bensoussan, D. Chang, H. Kiat, N. L. Klupp, J. P. Liu et al., "Chinese herbal medicines for people with impaired glucose tolerance or impaired fasting blood glucose," The Cochrane Database of Systematic Reviews, Article ID CD006690, 2009.

[3] E. S. Ford, G. Zhao, and C. Li, "Pre-diabetes and the risk for cardiovascular disease: a systematic review of the evidence," Journal of the American College of Cardiology, vol. 55, no. 13, pp. 1310-1317, 2010.

[4] Q. Jia, H. Zheng, X. Zhao et al., "Abnormal glucose regulation in patients with acute stroke across China: Prevalence and baseline patient characteristics," Stroke, vol. 43, no. 3, pp. 650-657, 2012.

[5] A. Karasik, "Glycaemic control is essential for effective cardiovascular risk reduction across the type 2 diabetes continuum," Annals of Medicine, vol. 37, no. 4, pp. 250-258, 2005.

[6] M. Alssema, J. M. Dekker, G. Nijpels, C. D. A. Stehouwer, L. M. Bouter, and R. J. Heine, "Proinsulin concentration is an independent predictor of all-cause and cardiovascular mortality: An 11-year follow-up of the Hoorn study," Diabetes Care, vol. 28, no. 4, pp. 860-865, 2005.

[7] Y.-X. Meng, E. S. Ford, C. Li et al., "Association of C-reactive protein with surrogate measures of insulin resistance among nondiabetic US from National Health and Nutrition Examination Survey 1999-2002," Clinical Chemistry, vol. 53, no. 12, pp. 2152-2159, 2007.

[8] S. E. Vermeer, W. Sandee, A. Algra, P. J. Koudstaal, L. J. Kappelle, and D. W. J. Dippel, "Impaired glucose tolerance increases stroke risk in nondiabetic patients with transient ischemic attack or minor ischemic stroke," Stroke, vol. 37, no. 6, pp. 14131417, 2006.

[9] M. M. Gabir, R. L. Hanson, D. Dabelea et al., "Plasma glucose and prediction of microvascular disease and mortality: evaluation of 1997 American Diabetes Association and 1999 World Health Organization criteria for diagnosis of diabets," Diabetes Care, vol. 23, no. 8, pp. 1113-1118, 2000. 
[10] Y. Mori, K. Hoshino, K. Yokota, T. Yokose, and N. Tajima, "Increased visceral fat and impaired glucose tolerance predict the increased risk of metabolic syndrome in Japanese middleaged men," Experimental and Clinical Endocrinology \& Diabetes: Official Journal, German Society of Endocrinology [and] German Diabetes Association, vol. 113, no. 6, pp. 334-339, 2005.

[11] T. Basturk and A. Unsal, "What is the frequency of carbohydrate metabolism disorder in CKD?" Journal of Renal Care, vol. 38, no. 1, pp. 15-21, 2012.

[12] Y. I. Kim, C.-H. Kim, C. S. Choi et al., "Microalbuminuria is associated with the insulin resistance syndrome independent of hypertension and type 2 diabetes in the Korean population," Diabetes Research and Clinical Practice, vol. 52, no. 2, pp. 145152, 2001.

[13] A. H. Xiang, R. K. Peters, S. L. Kjos et al., "Effect of pioglitazone on pancreatic $\beta$-cell function and diabetes risk in Hispanic women with prior gestational diabetes," Diabetes, vol. 55 , no. 2 , pp. 517-522, 2006.

[14] J.-L. Chiasson, R. G. Josse, R. Gomis, M. Hanefeld, A. Karasik, and M. Laakso, "Acarbose for prevention of type 2 diabetes mellitus: the STOP-NIDDM randomised trial," The Lancet, vol. 359, no. 9323, pp. 2072-2077, 2002.

[15] W. C. Knowler, E. Barrett-Connor, S. E. Fowler et al., "Reduction in the incidence of type 2 diabetes with lifestyle intervention or metformin," The New England Journal of Medicine, vol. 346, no. 6, pp. 393-403, 2002.

[16] Investigators TDT, "Effect of ramipril on the incidence of diabetes," The New England Journal of Medicine, vol. 35, no. 15, pp. 1551-1562, 2006.

[17] A. J. Scheen, "Drug treatment of non-insulin-dependent diabetes mellitus in the 1990s," Drugs, vol. 54, pp. 355-368, 1997.

[18] S. E. Nissen and K. Wolski, "Effect of rosiglitazone on the risk of myocardial infarction and death from cardiovascular causes," The New England Journal of Medicine, vol. 356, no. 24, pp. 24572471, 2007.

[19] H. J. H. Ai-zhen, L. Zhe-Feng, and C. Yuan, "Observation of clinical efficacy of Xiao-ke-hua-Yu-pian on impaired glucose tolerance," Medical Journal of Chinese People's Liberation Army, vol. 29, pp. 993-994, 2004.

[20] P. Shan-Yu, "Prevention and treating impaired glucose tolerance with Traditional Chinese Medicine," ZheJiang Journal of Integrated Traditional Chinese and Western Medicine, vol. 16, article 354, 2006.

[21] D.-H. D.-B. Wang and Z. Qi, "Observation of clinical efficacy of Traditional Chinese medicine on impaired glucose tolerance," Journal of Si Chuan of Traditional Chinese Medicine, vol. 24, pp. 63-64, 2006.

[22] X. Z. Wang, "Traditional Chinese medicine experience of professor TONG Xiao-lin in the treatment of steroid diabetes," Chinese Archives of Traditional Chinese Medicine, vol. 23, pp. 1764-1765, 2005.

[23] W.-Q. W.-H. Yu, "Observation of clinical efficacy of Jia-WeiYu-Ye-Tang on impaired glucose tolerance," Journal of ZheJiang University of Traditional Chinese Medicine, vol. 33, pp. 402-403, 2009.

[24] W. Yue-Xin, "Preventive effect of Jin-qi-jiang-tang-pian on impaired glucose tolerance," TianJin Medicial Journal, vol. 33, pp. 793-794, 2005.

[25] H. Shu-Ling, H. Shu-Fang, M. Ming, and Z. Yu-Qing, "Observation of Clinical Therapeutic Effects of Xiaodan Decoction on 32 Cases of Impaired Glucose Tolerance," World Journal of
Integrated Traditional and Western Medicine, vol. 2, article 016, 2009.

[26] X. Kai-Xia, Experimental study on preventive effect of tonifying kidney formulas fed during embryonic period on offspring rats' IGT which induced by high-fat diet Beijing University of Traditional Chinese Medicine, 2012.

[27] Y. Wang, Q. Feng, X. Niu et al., "The preventive effect of Zuogui wan on offspring rats' impaired glucose tolerance whose mothers had gestational diabetes mellitus," Evidence-Based Complementary and Alternative Medicine, vol. 2016, Article ID 9417362, 8 pages, 2016.

[28] H. Xu, J. Shen, H. Liu, Y. Shi, H. Li, and M. Wei, "Morroniside and loganin extracted from Cornus officinalis have protective effects on rat mesangial cell proliferation exposed to advanced glycation end products by preventing oxidative stress," Canadian Journal of Physiology and Pharmacology, vol. 84, no. 12, pp. 1267-1273, 2006.

[29] A. Y. Chen and Y. C. Chen, "A review of the dietary flavonoid, kaempferol on human health and cancer chemoprevention," Food Chemistry, vol. 138, no. 4, pp. 2099-2107, 2013.

[30] Q. J. Feng, M. L. Feng, and Y. L. Wang, "Effect of zuoguiwan on early embryonic development of mice," Zhongguo Zhong xi yi jie he za zhi Zhongguo Zhongxiyi jiehe zazhi= Chinese journal of integrated traditional and Western medicine/Zhongguo Zhong xi yi jie he xue hui, Zhongguo Zhong yi yan jiu yuan zhu ban, vol. 16, no. 11, pp. 673-675, 1996.

[31] A. Dobin, C. A. Davis, F. Schlesinger et al., "STAR: ultrafast universal RNA-seq aligner," Bioinformatics, vol. 29, no. 1, pp. 1521, 2013.

[32] M. Lawrence, W. Huber, H. Pags, P. Aboyoun, and M. Carlson, "Software for computing and annotating genomic ranges," PLoS Computational Biology, vol. 9, no. 8, Article ID e1003118, 2013.

[33] M. I. Love, W. Huber, and S. Anders, "Moderated estimation of fold change and dispersion for RNA-seq data with DESeq2," Genome Biology, vol. 15, no. 12, article 550, 2014.

[34] P. Shannon, A. Markiel, O. Ozier et al., "Cytoscape: a software Environment for integrated models of biomolecular interaction networks," Genome Research, vol. 13, no. 11, pp. 2498-2504, 2003.

[35] K. H. Moley, W. K. Vaughn, A. H. DeCherney, and M. P. Diamond, "Effect of diabetes mellitus on mouse pre-implantation embryo development," Journal of Reproduction and Fertility, vol. 93, no. 2, pp. 325-332, 1991.

[36] X.-M. Z.-Y. Luo, F. Yin, Y. Chen et al., "The effect of hyperglycemia on early mouse embryo development," Life Science Research, vol. 12, pp. 347-350, 2008.

[37] Z. Hong, Study on relationship between reactive oxygen, p66 (shc) and development block of mouse embryos induced by high glucose, Zhengzhou University, 2013.

[38] L. Zhang, Z.-S. Li, and N.-Z. Fang, "Glucose-induced ROS inhibits mouse early embryo development at late 2-cell stage in vitro," Academic Journal of Second Military Medical University, vol. 30, no. 8, pp. 888-891, 2009.

[39] K. D. Taylor and L. Pikó, "Expression of ribosomal protein genes in mouse oocytes and early embryos," Molecular Reproduction and Development, vol. 31, no. 3, pp. 182-188, 1992.

[40] M. Li-Li, Epigenetics research of abnormal glucose metabolism in uterus, Peking Union Medical College, 2011.

[41] C. E. H. Stewart and P. Rotwein, "Growth, differentiation, and survival: multiple physiological functions for insulin-like growth factors," Physiological Reviews, vol. 76, no. 4, pp. 10051026, 1996. 
[42] V. Rouiller-Fabre, L. Lecerf, C. Gautier, J. M. Saez, and R. Habert, "Expression and effect of insulin-like growth factor I on rat fetal Leydig cell function and differentiation," Endocrinology, vol. 139, no. 6, pp. 2926-2934, 1998.

[43] L. P. Liu, Y. L. Ren, and R. Li, "Effects of p38 signal pathway on osteoblastic cell differentiation by Zuogui pill medicated serum in MC3T3-E1 cell," Chinese Journal of Gerontology, vol. 7, article 042, 2013.

[44] C.-Y. Hao, Y.-L. Ren, and J.-R. Zhao, "Effects of Zuogui pill medicated serum via ERK/samds dependent pathway on MC3T3-E1 cell gene expression," Chinese Pharmacological Bulletin, vol. 28, no. 6, pp. 872-876, 2012.

[45] Z.-B. X.-C. Gong and G.-Q. Jin, "The study of modulating effect of zuogui pill on rat thymocytes apoptosis and expression of Bcl-2 and Bax," Chinese Journal of Gerontology, vol. 30, pp. 3290-3292, 2010.

[46] X.-F. Sun, L. Luo, Q.-C. Sun et al., "Experimental study of zuogui pills in stimulation of mouse immature oocytes nuclear maturation in vitro," Progress in Modern Biomedicine, vol. 3, article 015, 2011. 


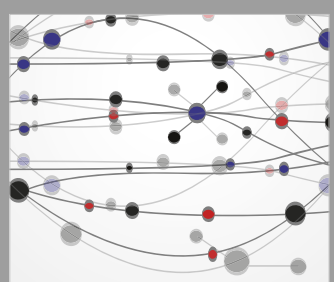

The Scientific World Journal
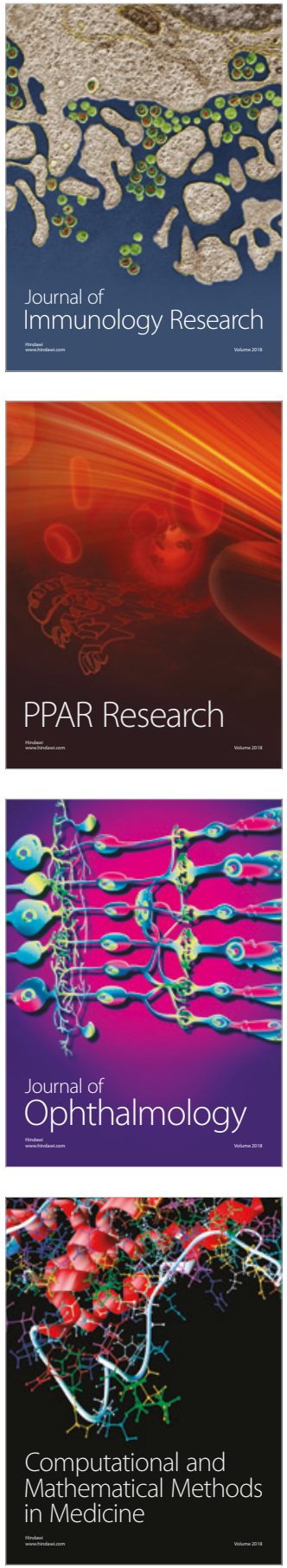

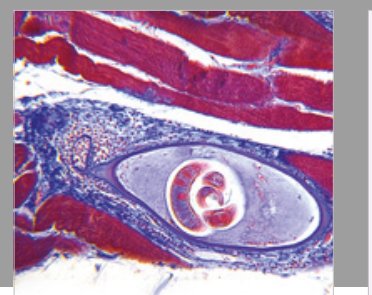

Gastroenterology Research and Practice

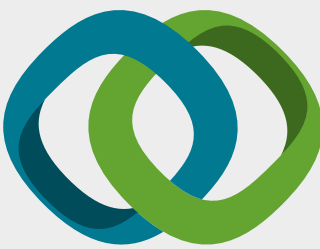

\section{Hindawi}

Submit your manuscripts at

www.hindawi.com
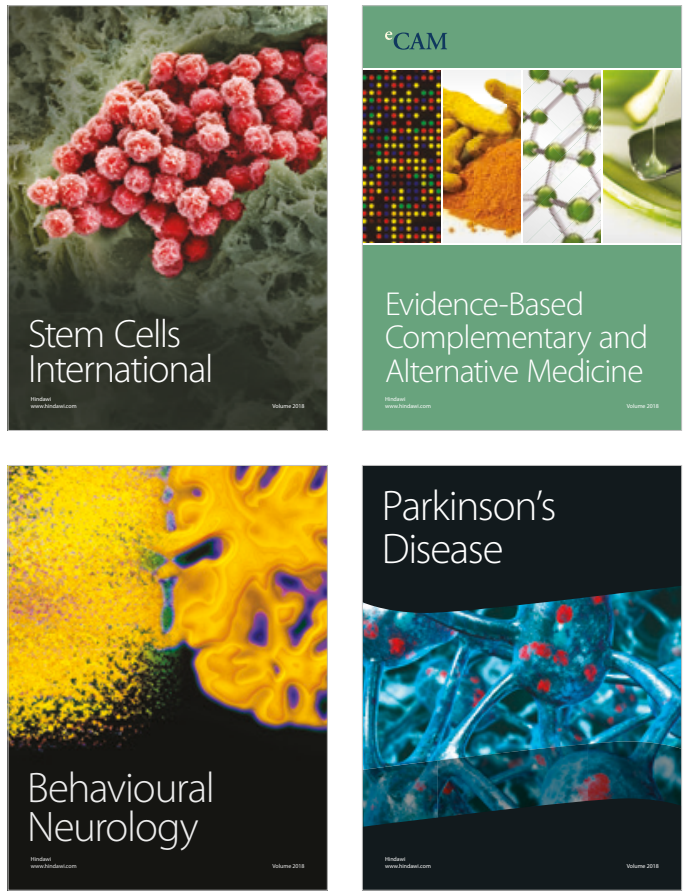

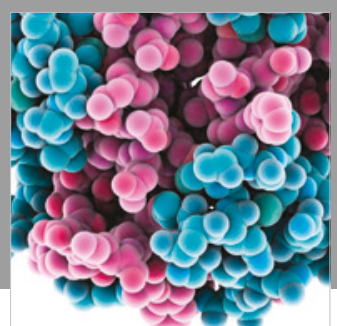

ournal of

Diabetes Research

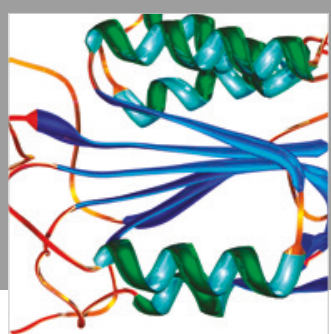

Disease Markers
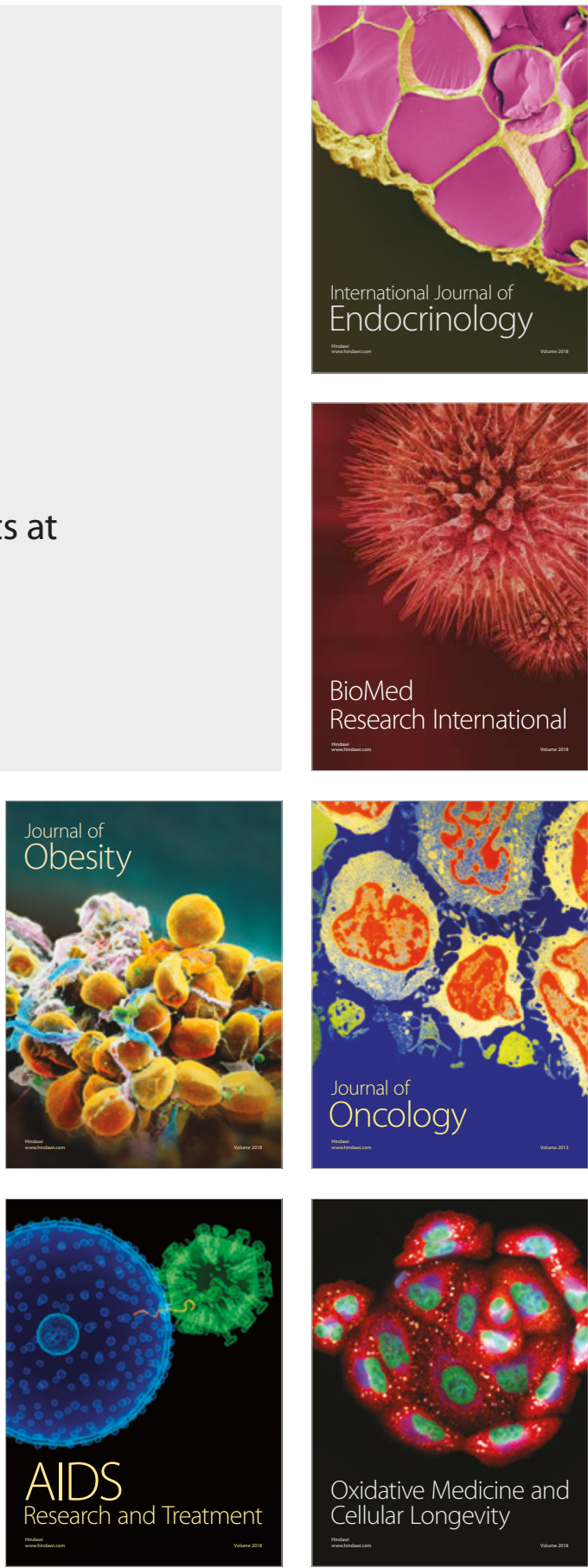